\title{
Correlation between cognition and plasma noradrenaline level in Alzheimer's disease: a potential new blood marker of disease evolution
}

\author{
Laure-Elise Pillet ${ }^{1}$, Camille Taccola', Justine Cotoni ${ }^{1}$, Hervé Thiriez ${ }^{1}$, Karine André ${ }^{2}$ and Romain Verpillot ${ }^{1}$
}

\begin{abstract}
Recent evidence showing degeneration of the noradrenergic system in the locus coeruleus (LC) in Alzheimer's disease (AD) has motivated great interest in noradrenaline (NA) as a potential brain hallmark of the disease. Despite the current exploration of blood markers for $A D$, the deregulation of the plasma NA concentration ( $[N A]_{\text {plasma }}$ ) in $A D$ is currently not well understood. This retrospective study includes a cohort of 71 patients (32 AD patients, 22 with other dementia and 17 without dementia) who were given consultations for memory complaints in the Cognitive Neurology Center of Lariboisière (Paris) between 2009 and 2014. As previously described in brain tissue, we show for the first time a linear correlation between $[N A]_{\text {plasma }}$ and Mini Mental State Examination (MMSE) score in AD patients. We observed that high $[N A]_{\text {plasma }}$ in $A D$ patients was associated with higher $\left[A \beta_{1-42}\right]_{C S F}$ than in other AD patients with $[N A]_{\text {plasma }}$ similar to NC patients. In parallel, we observed a lower (p-Tau/Tau) $)_{C S F}$ in $A D$ patients with low $[N A]_{\text {plasma }}$ than in non-AD patients with $[\mathrm{NA}]_{\text {plasma }}$ similar to $[\mathrm{NA}]_{\text {plasma }}$ in $\mathrm{NC}$ patients. Our data suggest that $[\mathrm{NA}]_{\text {plasma }}$ could be a potential biomarker of disease evolution in the context of $A D$ and could possibly improve early diagnosis.
\end{abstract}

\section{Introduction}

Alzheimer's disease (AD) is the most common cause of dementia among adults over 65 years old and is characterized by progressive impairment in cognitive function and behavior ${ }^{1}$. The neuropathological hallmarks of the disease include amyloid plaques, formed by extracellular accumulation of amyloid $\beta$ peptide $\left(\mathrm{A} \beta_{1-42}\right)$ and neurofibrillary tangles, formed by intraneuronal aggregates of phosphorylated Tau protein p-181 (p-Tau $)^{2}$. Cerebrospinal fluid (CSF) core biomarkers $\left(\mathrm{A} \beta_{1-42}, \mathrm{p}\right.$-Tau, and total-Tau), as well as amyloid and Tau positron-emission tomography, reliably reflect AD neuropathological brain lesions ${ }^{3,4}$, leading to their inclusion in research diagnostic criteria. However, acquiring these measurements is costly and invasive. In addition, CSF biomarkers are sometimes not sufficient to differentiate $\mathrm{AD}$ from other types of

Correspondence: Romain Verpillot (romain.verpillot@alzohis.com)

${ }^{1}$ Alzohis, 28 Rue du Faubourg Poissonnière, 75010 Paris, France

${ }^{2}$ Statitec, Groupe MultiHealth, Vélizy Espace-Immeuble Santos Dumont, 13

Avenue Morane Saulnier, 78140 Vélizy, Villacoublay, France dementia, such as dementia with Lewy bodies ${ }^{5}$. Indeed, differential diagnosis remains a major challenge in clinical practice $^{6}$. Thus, addressing the need to develop novel and inexpensive diagnostic techniques has become a focal point of Alzheimer's research ${ }^{2}$.

\section{The noradrenergic system and AD}

Many studies have shown that the abnormal structure and function of noradrenergic neurons are closely related to $\mathrm{AD}$ pathophysiology ${ }^{6-8}$. The noradrenergic system plays pivotal regulatory roles in various behaviors, including selective attention, memory storage and retrieval, vigilance, and $\operatorname{mood}^{9}$. Most of the noradrenergic neurons in the CNS originate from the locus coeruleus (LC) and project to different areas of the brain, such as the cortex, hippocampus, amygdala, thalamus, and hypothalamus ${ }^{10}$. Hyperphosphorylation of Tau protein in the LC appears at an early stage of $\mathrm{AD}$ pathogenesis ${ }^{11}$, and the number of neurons in the LC has been shown to progressively decrease during the disease, beginning at the 
prodromal stage of $\mathrm{AD}^{8,12-17}$. Based on the current literature, one of the ideas that has emerged is that the onset of $\mathrm{AD}$ is preceded by abnormal hyperactivation of the $\mathrm{LC}$, resulting in oversecretion of NA in the $\operatorname{cortex}^{18}$. The hyperactivation of noradrenergic receptors in the cortex leads to a cortical accumulation of $A \beta_{1-42}$ plaques $^{19}$. Following this accumulation, the dendritic spine density of noradrenergic neurons and noradrenaline (NA) secretion begin to decrease due to lack of stimulation and/or (glial) inflammatory processes ${ }^{18}$. However, this working hypothesis requires further research in this area and supporting evidence. NA is a catecholamine that acts as a neuromodulator when synthesized by noradrenergic neurons and as a hormone when synthesized by the sympathetic system. NA deregulation in $A D$ was first identified at the end of the 1970s by Adolfsson et al., who showed that the NA concentration in postmortem brain tissue was lower in $\mathrm{AD}$ patients of $\sim 75$ years of age than in age-matched controls and correlated with dementia score $^{20}$. Interestingly, a recent publication reported a correlation between the NA concentration in a specific cortical brain region of AD patients and MMSE score ${ }^{21}$. In addition, NA CSF levels in advanced AD patients differ from those in patients with mild to moderate severity and those in healthy older subjects ${ }^{22,23}$. These observations imply that cognitive decline could be timely correlated with coeruleo-cortical network deregulation and disconnection ${ }^{18}$. The early increase in neuronal NA secretion before the onset of $\mathrm{AD}$ that is later followed by noradrenergic neuron degeneration could be related to disease evolution ${ }^{11,24}$. At the peripheral level, NA circulates through the bloodstream and is known to be involved in the body response to acute stress by modulating, among other physiological parameters, heart rate, blood pressure, and muscle contraction ${ }^{25}$. Interestingly, the LC is known to regulate sympathetic activity ${ }^{26}$ by modulating postsympathetic noradrenergic neurons targeting the heart and blood vessels ${ }^{27}$. Different studies have shown that the plasma NA concentration ( $\left.[\mathrm{NA}]_{\text {plasma }}\right)$ is altered in AD. Although these studies show conflicting results, it seems clear that $\mathrm{AD}$ pathophysiology is associated with modulations in $[\mathrm{NA}]_{\text {plasma }}{ }^{23,28-30}$.

\section{Early stage of AD: CSF and plasma biomarkers}

Investigations of potential treatments for $\mathrm{AD}$ have revealed the need to define and characterize an early stage of the disease, where therapeutic treatments could be the most effective. Recent studies have attempted to identify new biomarkers that can identify $\mathrm{AD}$ patients before the onset of advanced symptoms. The term mild cognitive impairment (MCI) was used for the first time in the 1980s to define patients at an intermediate stage between normal aging patients and patients with dementia ${ }^{31}$. Recently, the National Institute on Aging and Alzheimer's
Association (NIA-AA) specified the state "MCI due to $A D$ " to clearly identify a very early stage of this disease ${ }^{32}$. Among other clinical criteria $^{33}$, CSF biomarkers are required in the determination of $\mathrm{MCI}$ due to $\mathrm{AD}$, such as CSF $\mathrm{A} \beta_{1-42}$, total-Tau, and/or $\mathrm{p}$-Tau concentrations ${ }^{32}$. Several studies in the literature have shown a significant difference in CSF $\mathrm{A} \beta_{1-42}{ }^{34-36}$, total-Tau ${ }^{35-39}$, and p-Tau ${ }^{37}$ concentrations between $\mathrm{MCI}$ and $\mathrm{AD}$ patients, describing a decrease in the CSF $A \beta_{1-42}$ concentration ( $\left[\mathrm{A} \beta_{1-42}\right]_{\mathrm{CSF}}$ ) and an increase in the CSF total-Tau and p-Tau concentrations $\left([\mathrm{Tau}]_{\mathrm{CSF}}\right.$ and $\left.[\mathrm{p}-\mathrm{Tau}]_{\mathrm{CSF}}\right)$ in $\mathrm{AD}$ patients. However, these differences are not always statistically significant for CSF $A \beta_{1-42}{ }^{37,40}$, total-Tau ${ }^{34,40}$, and $\mathrm{p}-\mathrm{Tau}^{34,35,39,40}$. A higher CSF p-Tau/total Tau ratio ( $\mathrm{Tau} / \mathrm{Tau})_{\mathrm{CSF}}$ ) (or lower $\left.(\mathrm{Tau} / \mathrm{p}-\mathrm{Tau})_{\mathrm{CSF}}\right)$ was also previously described in $\mathrm{AD}$ patients in comparison with healthy controls ${ }^{41,42}$. Furthermore, $(\mathrm{p}-\mathrm{Tau} / \mathrm{Tau})_{\mathrm{CSF}}$ has been shown to be lower in AD patients than in control patients and patients with fronto-temporal dementia ${ }^{43}$. However, no difference in $(\mathrm{p}-\mathrm{Tau} / \mathrm{Tau})_{\mathrm{CSF}}$ was found between patients with mild AD and those with moderate $\mathrm{AD}^{41}$. (p-Tau/Tau $)_{\mathrm{CSF}}$ was also associated with the rate of cognitive decline, showing a negative dose-dependent relation between $(\mathrm{p}-\mathrm{Tau} / \mathrm{Tau})_{\mathrm{CSF}}$ and yearly MMSE score fold change during $\mathrm{AD}^{44}$. In other words, a low (p-Tau/ $\mathrm{Tau})_{\mathrm{CSF}}$ in patients with $\mathrm{AD}$ is associated with a faster cognitive decline than that observed in other AD patients with higher $(\mathrm{p}-\mathrm{Tau} / \mathrm{Tau})_{\mathrm{CSF}}$. This association implies that $\mathrm{AD}$ patients with low $\left[\mathrm{A} \beta_{1-42}\right]_{\mathrm{CSF}}$ and elevated $[\mathrm{Tau}]_{\mathrm{CSF}}$ and $[\mathrm{p}-\mathrm{Tau}]_{\mathrm{CSF}}$ are more likely to be in an advanced stage of the disease.

Although these results are conflicting, the CSF AD biomarker profile seems helpful in the differentiation of $\mathrm{MCI}$ patients from $\mathrm{AD}$ patients and provides information about whether the patients is in an early or late stage of the disease.

The need to find alternative body fluids for biomarker identification in $\mathrm{AD}$ has recently led investigators to analyze currently known CSF biomarkers for $\mathrm{AD}$ in plasma ${ }^{45-57}$. Studies in this area have shown conflicting results with (i) no difference between $\mathrm{AD}$ and control patients ${ }^{47,49,50}$, (ii) differences in plasma $\mathrm{A} \beta_{1-42}{ }^{45,58}$, $\mathrm{Tau}^{57}$ and $\mathrm{p}-\mathrm{Tau}^{54}$ concentrations between $\mathrm{AD}$ and control patients, and (iii) differences in plasma $\mathrm{A} \beta_{1-42}{ }^{53,58}$, Tau, and $\mathrm{p}-\mathrm{Tau}^{54}$ levels among patients with MCI due to AD, patients with $\mathrm{AD}$, and control subjects. Other articles have reported that the plasma $A \beta_{1-42}$ concentration might change during disease evolution, showing abnormally high concentrations at the preclinical stage or beginning of cognitive decline that decrease progressively during $A D$ $\operatorname{progression}^{51,55}$. Moreover, other studies have shown different linear correlations in the context of $\mathrm{AD}$ (i) between imaging data of amyloid plaque deposition in the brain and $\operatorname{CSF}^{59}$ or plasma ${ }^{46,48}$ biomarker concentrations 
and (ii) between CSF and plasma biomarker concentrations ${ }^{45,47,50,57,58}$. The mechanisms behind brain AD biomarker clearance pathways (blood-brain, CSF-brain and blood-CSF barriers) are not yet understood, and it is not clear if those barriers are altered during the disease. Nonetheless, some articles suggest that biomarkers in plasma could mirror brain and CSF biomarker deposition and metabolism ${ }^{60}$. However, some studies did not observe any correlation between CSF and plasma biomarker concentrations ${ }^{56}$. There are a variety of possible factors that could explain the inconsistent conclusions and concentration value heterogeneity among studies. For example, technical (antibody used, time and temperature of sample conservation, hydrophobic and albumin binding properties of $A \beta$, low concentration in comparison with CSF, etc.) and physiological (effect of age and diet, peripheral origins, kidney and hepatic clearance, etc.) reasons $^{51,58}$ may make determining plasma $A \beta_{1-42}$ level alterations in the context of $\mathrm{AD}$ difficult. For these reasons, the clinical utility of plasmatic $A \beta_{1-42}$ and Tau has not yet been demonstrated, and the need to identify other plasma $\mathrm{AD}$ biomarkers is crucial for $\mathrm{AD}$ diagnosis.

In this retrospective study, we explored $[\mathrm{NA}]_{\text {plasma }}$ in patients between 58 and 79 years old consulting for memory complaints for the first time. Our study examined the relationship between $[\mathrm{NA}]_{\text {plasma }}$ and concomitant diagnostic criteria such as MMSE score and CSF biomarker profile $\left(\mathrm{A} \beta_{1-42}\right.$, Tau and $\left.\mathrm{p}-\mathrm{Tau}\right)$. Due to the deregulation of noradrenergic transmission in the brain during $\mathrm{AD}$, we wanted to determine whether $[\mathrm{NA}]_{\text {plasma }}$ could be correlated with clinical parameters reflecting the stage of the disease at the cognitive (MMSE score) and molecular ( $\mathrm{A} \beta_{1-42}$, Tau, and $\mathrm{p}$-Tau CSF biomarkers) levels.

\section{Materials/subjects and methods Study population}

All patients presented to the Cognitive Neurology Center of Lariboisière (Paris) for their first consultation between 2009 and 2014. Patients involved in this study were between 58 and 79 years old at the time of blood sampling. MMSE score and lumbar puncture were performed the day of blood sampling or $<1$ month later. The MMSE is a worldwide commonly used, easy to apply and rapid screening tool lasting $<10 \mathrm{~min}$. The MMSE score provides supporting information for dementia diagnosis that evaluates global cognitive impairment for all causes of dementia ${ }^{61}$. Briefly, the paper-based test consists of 11 orally administered questions with verbal and written responses concerning different domains (attention, memory, orientation, language, and ability to follow verbal and written commands) with a maximum score of 30 (normal cognition) and lower scores highlighting a severe deficit $^{62}$. The original article published in 1975 defined 24 as a cutoff score for normal cognition with a sensitivity and specificity of $87 \%$ and $82.6 \%$, respectively ${ }^{61}$. In a more recent study, the mean MMSE score of the population older than 90 years without dementia was reported to be 26.6, whereas the cutoff score for normal cognition was reported to be 23.3, very similar to the cutoff score for normal cognition in the younger population ( 23 or 24 points $)^{62,63}$. In the context of AD, the MMSE score is used to assess disease severity and is occasionally used to estimate efficacy in clinical drug trials ${ }^{64}$. Previous studies have shown that MMSE scores decrease during AD progression at different rates depending on disease progression and patient education ${ }^{65}$. More generally, patients in the moderate to severe stages of AD have an MMSE score below $20^{66}$. Sample size was calculated based on results from previous articles highlighting a significant difference in plasma catecholamines between $\mathrm{AD}$ and non- $\mathrm{AD}$ patients. Raskind et al. ${ }^{23}$ observed a significant difference between advanced $\mathrm{AD}$ and control patients with a small sample size ( $<20$ patients). Therefore, we based our calculation for sample size on the article of Umageki et $\mathrm{al}^{28}$ that examined adrenaline levels. Considering a common standard deviation of $18.46 \mathrm{pg} / \mathrm{mL}$, a sample size of 68 patients is necessary to show a difference of $12.69 \mathrm{pg} / \mathrm{mL}$ between the two groups with a power of $80 \%$ and an $\alpha$ of $5 \%$. Seventy-one patients were included in this retrospective study: $32 \mathrm{AD}$ patients (diagnoses were performed according to NIA-AA guidelines ${ }^{4}$ ), 22 other dementia (OD; frontotemporal dementia, vascular dementia, or dementia with Lewy bodies) patients, and 17 neurological control (NC) patients. NC patients were defined as those with memory complaints, mental depression or anxiety but for whom no dementia was diagnosed. The following cutoff values for core AD CSF biomarkers were used as supportive criteria for dementia due to AD: A $\beta 1-42(<550 \mathrm{pg} / \mathrm{mL})$, total-Tau $(>400 \mathrm{pg} /$ $\mathrm{mL})$, and $\mathrm{p}-\mathrm{Tau}(>50 \mathrm{pg} / \mathrm{mL})$. Demographic information, MMSE scores, presence of the APOE $\varepsilon 4$ allele, CSF biomarkers $\left(\mathrm{A} \beta_{1-42}\right.$ protein, total Tau, and $\mathrm{p}$-Tau protein) and concomitant medications of the studied cohort are shown in Table 1.

\section{Plasma NA quantification}

Patients fasted overnight (for $\sim 12 \mathrm{~h}$ ) before blood collection and were in the decubitus position during sampling. Plasma samples were purified and analyzed with a reagent kit for HPLC analysis of catecholamines in plasma (Chromsystems, order \#5000) according to the manufacturer's instructions. Briefly, blood samples were stabilized with glutathione, and plasma was isolated less than $1 \mathrm{~h}$ after blood sampling by centrifugation. Plasma samples were stored at $-80^{\circ} \mathrm{C}$. After thawing, $1 \mathrm{~mL}$ of plasma was used to extract catecholamines for dosage by high-performance liquid chromatography coupled with 
Table 1 Demographic and physiologic data of studied cohort.

\begin{tabular}{|c|c|c|c|c|c|}
\hline & & NC & OD & $A D$ & $p$ value \\
\hline \multicolumn{2}{|l|}{ Total number of patients } & 17 & 22 & 32 & - \\
\hline Sex & $\%$ of female patients & 52.9 & 36.4 & 56.3 & 0.3360 \\
\hline Age & Age mean (SD) in year & $67(6.671)$ & $67.27(5.650)$ & $70.69(6.703)$ & 0.0746 \\
\hline MMSE & MMSE score median (IQR) & $28(27-29)$ & $21(16.25-23.25)$ & $23(20-25)$ & $<0.0001$ \\
\hline$A P O E 4^{\mathrm{a}}$ & $\%$ of patients carrying APOE $\varepsilon 4$ allele & 15.4 & 36.8 & 70.0 & 0.0021 \\
\hline CSF $A \beta_{1-42}$ concentration $^{\mathrm{b}}$ & CSF A $\beta$ concentration mean (SD) & $795.4(155,8)$ & $793.1(294.5)$ & $419.2(162.2)$ & $<0.0001$ \\
\hline CSF Tau concentration ${ }^{\text {b }}$ & CSF Tau concentration median (IQR) & $172(139-239.5)$ & $245(190-299)$ & $581.5(388.8-766.8)$ & $<0.0001$ \\
\hline CSF p-Tau concentration ${ }^{b}$ & CSF p-Tau concentration median (IQR) & $34.50(19.50-44.75)$ & $44(34-59.50)$ & $88.5(69.45-113.50)$ & $<0.0001$ \\
\hline Plasma NA concentration & Plasma NA concentration median (IQR) & $2564(1614-3131)$ & $2108(1540-2561)$ & $2194(1846-3534)$ & 0.3873 \\
\hline \multirow[t]{7}{*}{$\begin{array}{l}\% \text { of patients with co- } \\
\text { medication }\end{array}$} & $\begin{array}{l}\text { Anti-Alzheimer or anti-Parkinsonian/ } \\
\text { dopaminergic agents }\end{array}$ & 5.9 & 22.7 & 18.8 & 0.3536 \\
\hline & Antidepressants & 23.5 & 27.3 & 21.9 & 0.9000 \\
\hline & $\begin{array}{l}\text { Benzodiazepines (anxiolytics/hypnotics) and } \\
\text { Neuroleptics }\end{array}$ & 5.9 & 18.2 & 9.4 & 0.4361 \\
\hline & Lipid-lowering agents, oral antidiabetics & 35.3 & 40.9 & 28.1 & 0.6143 \\
\hline & Anti-hypertensive agents & 52.9 & 31.8 & 31.3 & 0.2770 \\
\hline & Veinotonics / vasodilatators & 0.0 & 0.0 & 0.0 & - \\
\hline & $\begin{array}{l}\text { Others (Vitamines, anti-asthmatics, non steroidal } \\
\text { anti-inflammatory agents) }\end{array}$ & 23.5 & 18.2 & 12.5 & 0.6069 \\
\hline
\end{tabular}

${ }^{a}$ Five $N C$, three $O D$, and two $A D$ patients did not undergo APOE genotyping.

${ }^{\mathrm{b}}$ Three $\mathrm{NC}$, one $\mathrm{OD}$, and two $\mathrm{AD}$ patients did not undergo lumbar puncture.

electrochemical detection. Experimenters did not know the corresponding group of the sample during dosage.

\section{CSF biomarker quantification}

Lumbar punctures were performed on fasting patients, typically between 9 and 12 a.m. CSF samples were centrifuged at $1 \mathrm{~g}$ for $10 \mathrm{~min}$ at $4{ }^{\circ} \mathrm{C}$ within $4 \mathrm{~h}$ of collection, aliquoted in $0.5-\mathrm{mL}$ polypropylene tubes and stored at $-80^{\circ} \mathrm{C}$ for further analysis. CSF levels of $\mathrm{A} \beta_{1-42}$, total Tau, and $\mathrm{p}$-Tau were measured using the commercially available sandwich ELISA INNOTEST ${ }^{\circledR}$, according to the manufacturer's procedures (Fujirebio Europe NV, formerly Innogenetics NV).

\section{Statistical analysis}

Depending on the normality of the data (D'AgostinoPearson normality test), the results are presented as the mean with standard deviation (SD) (standard error of mean in figures) or median with interquartile range (IQR: 25-75th percentiles) (95\% confidence interval in figures). For normally distributed data, we performed Student's $t$ test (or Student's $t$ test with Welch's correction if the F-test showed significantly different variances between groups) or one-way ANOVA. For non-normally distributed samples, we performed a Mann-Whitney test or Kruskal-Wallis test. Fisher's exact test was used to compare frequencies. Linear correlation coefficients were calculated using Pearson's correlation test or Spearman's correlation test for normally or not normally distributed data, respectively. To compare distributions of data, we performed the Kolmogorov-Smirnov test. We performed Rosner's Extreme Studentized Deviate test (using lognormal distribution for plasma and CSF biomolecules) for multiple outliers (two-sided test) with a $p$ value of 0.01 , and we found no outliers for MMSE score, $[\mathrm{NA}]_{\text {plasma, }}$ $\left[\mathrm{A} \beta_{1-42}\right]_{\mathrm{CSF}},[\mathrm{Tau}]_{\mathrm{CSF}}$, or $[\mathrm{p}-\mathrm{Tau}]_{\mathrm{CSF}}$. Analyses were performed using GraphPad Prism 8.0.1 software. Statistical significance was set at $p$ value $<0.05$.

\section{Results}

\section{Characterization of the study cohort}

The studied groups did not significantly differ by sex ratio, age, or concomitant treatments (Table 1). As expected, they differed by MMSE score and by APOE $\varepsilon 4$ carrier status (Table 1). Clinical diagnosis of $\mathrm{AD}$ made by the neurologist was based on age, MMSE score, and CSF biomarkers, according to NIA-AA guidelines ${ }^{4}$. AD patients had significantly lower $A \beta_{1-42}$, higher $\mathrm{p}-\mathrm{Tau}$, and 
higher total-Tau CSF concentrations than OD and NC patients (Table 1).

\section{Correlation between plasma NA concentration and cognitive MMSE score in AD patients}

As previously described in a specific cortical brain region $^{21}$, we observed a significant linear correlation between $[\mathrm{NA}]_{\text {plasma }}$ at the peripheral level and MMSE score in AD patients (Spearman's correlation, $r=0.4426$ (95\% CI: 0.1004-0.6912); $p$ value $=0.0112$; equation: $Y=$ 126.3* $X+158$ ) (Fig. 1a). However, this correlation was not observed in non-AD patients (Spearman's correlation, $r=-0.01385$ (95\% $\mathrm{CI}:-0.3365$ to 0.3117 ); $p$ value $=0.9333$; equation: $Y=-44.63^{*} X+3556$ ) (Fig. $1 \mathrm{~b})$. These results suggest a possible link between $[\mathrm{NA}]_{\text {plasma }}$ and cognitive decline in $\mathrm{AD}$ patients. Nonetheless, we observed no difference in $[\mathrm{NA}]_{\text {plasma }}$ among the AD, OD and NC patients (Table 1). We discriminated patients with an MMSE score above $(\geq)$ and below $(<)$ 24, which correspond to the cutoff score for normal cognition in the literature ${ }^{67}$. In non-AD patients, we observed no difference in the distribution of $[\mathrm{NA}]_{\text {plasma }}$ between patients with an MMSE score above and those with a score below 24 (Kolmogorov-Smirnov test, $p$ value $=$ 0.7459) (Fig. 2a). On the other hand, we found a significant difference between the distribution of $[\mathrm{NA}]_{\text {plasma }}$ in $\mathrm{AD}$ patients with an MMSE score above and those with a score below 24 (Kolmogorov-Smirnov test, $p$ value $=$ 0.0260) (Fig. 2b). Moreover, we observed that the median $[\mathrm{NA}]_{\text {plasma }}$ of AD patients with an MMSE score above 24 was significantly higher than the median $[\mathrm{NA}]_{\text {plasma }}$ of non-AD patients with a similar MMSE score $(\geq 24)$ (Mann-Whitney test, $p$ value $=0.0287$ ) and lower $(<24)$ MMSE score (Mann-Whitney test, $p$ value $=0.0136)$ and than the median $[\mathrm{NA}]_{\text {plasma }}$ of other $\mathrm{AD}$ patients (Mann-Whitney test, $p$ value $=0.0177$ ). We observed no difference between the median $[\mathrm{NA}]_{\text {plasma }}$ of AD patients with an MMSE score below 24 and that of non-AD patients with similar (Mann-Whitney test, $p$ value = 0.8757 ) or higher (Mann-Whitney test, $p$ value $=0.3851$ ) MMSE scores (Fig. 2c). Taken together, these results support the idea that there is a subpopulation of $A D$ patients with an MMSE score above 24 and a higher median $\{\mathrm{NA}]_{\text {plasma }}$ than other patient groups, implying that a mild cognitive decline in AD depicted by MMSE score is associated with an elevated $[\mathrm{NA}]_{\text {plasma. }}$.

\section{NA plasma level in AD is associated with MMSE score and CSF biomarker profile}

To determine whether $[\mathrm{NA}]_{\text {plasma }}$ reflects early disease evolution, we examined whether there was a correlation between the distance from the $\mathrm{NC}$ patient median $[\mathrm{NA}]_{\text {plasma }}$ and the CSF biomarker profile. Because of the non-normal distribution of $[\mathrm{NA}]_{\text {plasma }}$ data, we decided to

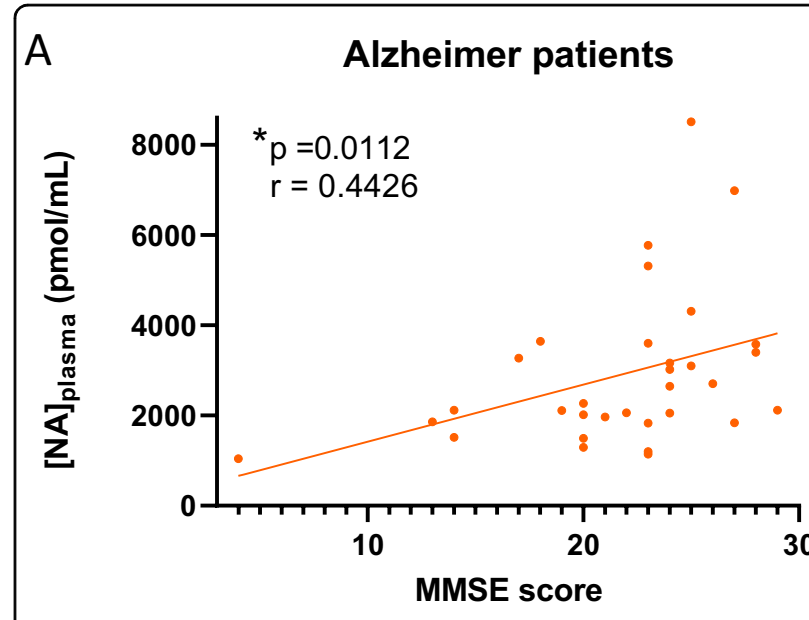

B non-Alzheimer patients

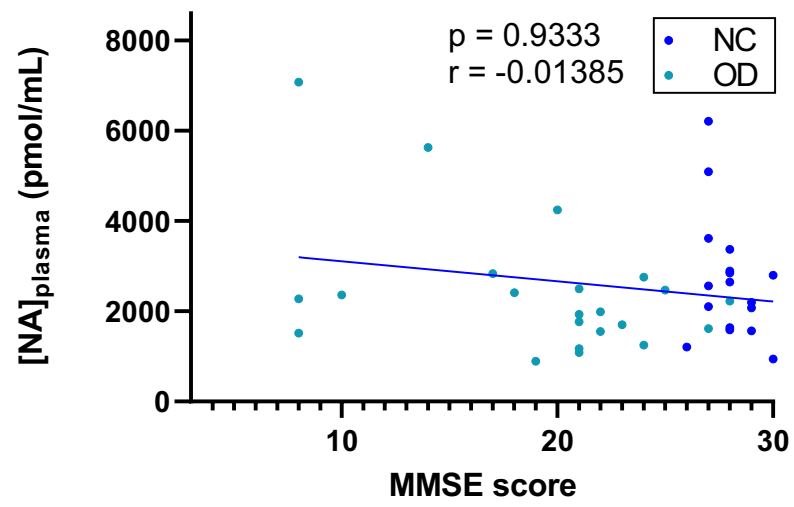

Fig. 1 MMSE score correlates with $[N A]_{\text {plasma }}$ in AD patients. Linear regression between $[N A]_{\text {plasma }}$ and MMSE score of $A D(\mathbf{a})$ and non-AD (b) patients. * indicates $p$ value $<0.05$.

use logarithmic data as previously described ${ }^{68}$ (in the following text, all statistical tests for CSF biomarkers were performed on logarithmic data). This distance was defined as the absolute value of $\ln (\mathrm{NA})_{\text {plasma }}-<\ln (\mathrm{NA})_{\text {plasma/N }}>$, with $<\ln (\mathrm{NA})_{\text {plasma/N }}>$ the median value of logarithmic $[\mathrm{NA}]_{\text {plasma }}$ from $\mathrm{NC}$ patients $\left(<\ln (\mathrm{NA})_{\text {plasma/NC }} \geq 7.849\right)$. We found a significant positive linear correlation between this absolute distance and $\left[\mathrm{A} \beta_{1-42}\right]_{\mathrm{CSF}}$ in $\mathrm{AD}$ patients (Pearson's correlation, $r=0.3694$ (95\% CI 0.01047 to $0.6439) ; \quad p$ value $=0.0446$; equation: $Y=0.6449 * X+$ 5.695) (Fig. 3a), but this correlation was not observed in non-AD patients (Pearson's correlation, $r=-0.1629(95 \%$ CI -0.4706 to 0.1801$) ; p$ value $=0.3498$; equation: $Y=$ $-0.1662 * X+6.697$ ) (Fig. 3b). Looking at the relative distance of $\ln [\mathrm{NA}]_{\text {plasma }}$ from $<\ln (\mathrm{NA})_{\text {plasma/NC }}>$ (negative or positive when respectively lower or higher than the $\mathrm{NC}$ patients median), we observed a negative correlation that does not reach significance for negative distance 




B Alzheimer patients

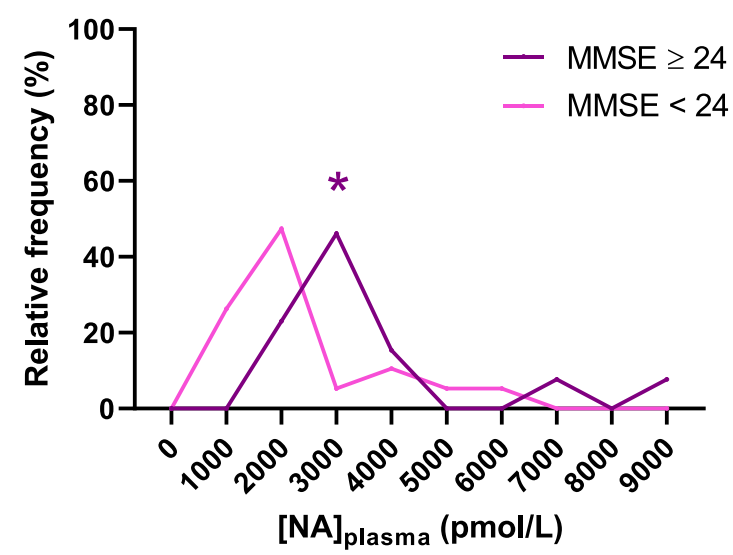

C

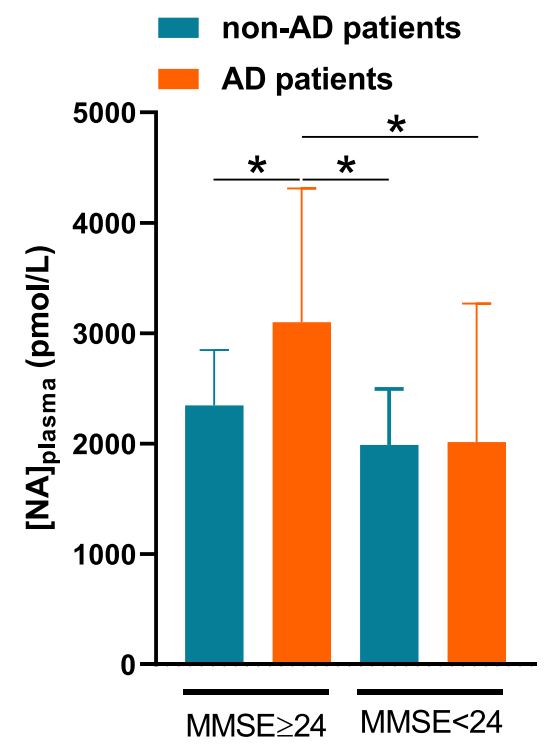

Fig. 2 Existence of subpopulations of AD patients described by MMSE score and $[\mathrm{NA}]_{\text {plasma. }}$ a Relative frequency distribution of $[N A]_{\text {plasma }}$ in non-AD patients with MMSE score above and below 24. b Relative frequency distribution of $[N A]_{\text {plasma }}$ in $A D$ patients with MMSE score above and below 24. c [NA $]_{\text {plasma }}$ median of NC, OD, and AD patients with MMSE score above and below $24 .{ }^{*}$ indicates $p$ value $<0.05$.
(Pearson's correlation, $r=-0.2094$ (95\% CI -0.6160 to $0.2854) ; \quad p$ value $=0.4044$; equation: $Y=-0.3175^{*} X+$ 5.786) and a significant positive correlation for positive distance (Pearson's correlation, $r=0.6212 \quad(95 \%$ CI: $0.1352-0.8663) ; p$ value $=0.0177$; equation: $Y=1.192 * X+$ 5.499) (Fig. 3c). Similarly, we found a negative linear correlation between absolute distance from $\left\langle\ln (\mathrm{NA})_{\text {plasma/ } / \mathrm{N}}>\right.$ and (p-Tau/Tau) $)_{\mathrm{CSF}}$ in AD patients (Pearson's correlation, $r=-0.3907$ (95\% CI: -0.6583 to -0.03541$) ; p$ value $=$ 0.0328; equation: $Y=-0.3079^{*} X-1.687$ ) (Fig. 3d) but not in non-AD patients (Pearson's correlation, $r=-0.2385$ (95\% CI: -0.5296 to 0.1029$)$; $p$ value $=0.1677$; equation: $Y=-0.1737^{*} X-1.621$ ) (Fig. 3e). An examination of the relative distance from $<\ln (\mathrm{NA})_{\text {plasma/NC }}>$ revealed a significant positive correlation for negative distance (Pearson's correlation, $r=0.5605$ (95\% CI: $0.1269-0.8143) ; p$ value $=$ 0.0155 ; equation: $\left.Y=0.4962^{*} X-1.625\right)$ and a negative correlation for positive distance that does not reach significance (Pearson's correlation, $r=-0.2346$ (95\% CI: -0.6805 to 0.3380$) ; p$ value $=0.4194$; equation: $Y=$ $-0.1361^{*} X-1.732$ ) (Fig. 3f). Taken together, our results suggest that $[\mathrm{NA}]_{\text {plasma }}$ may reflect the progression of $\mathrm{AD}$, highlighting the existence of different groups of $\mathrm{AD}$ patients characterized by different biomarkers.

\section{Discussion}

There is a significant amount of literature on deregulation of cerebral, plasma, and urine NA concentrations in murine models of $\mathrm{AD}^{69-72}$ and in human $\mathrm{AD}$ patients $^{21,28,29,73,74}$. In mouse models, $\mathrm{NA}$ has been implicated in the microglial phagocytosis of $A \beta_{1-42}$ plaques and cognition ${ }^{24,75}$. In humans, NA levels in cortical brain regions have been correlated with dementia score ${ }^{20}$ and MMSE score ${ }^{21}$, which decreases during AD development ${ }^{67}$. This observation is consistent with our results showing a significant correlation between $[\mathrm{NA}]_{\text {plasma }}$ and MMSE score in $\mathrm{AD}$ patients. Regarding those results, it is tempting to say that $[\mathrm{NA}]_{\text {plasma }}$ could be a mirror of cerebral NA deregulation and could help in the diagnosis of AD. Previous studies have shown an increase ${ }^{23}$ or a decrease $^{28}$ in $[\mathrm{NA}]_{\text {plasma }}$ in $\mathrm{AD}$ patients in comparison with the concentration in control patients. This discordance could be explained by the existence of subpopulations of $\mathrm{AD}$ patients, as suggested previously $\mathrm{y}^{23}$ and as identified in our analysis as patients with a high distance from the median $[\mathrm{NA}]_{\text {plasma }}$ of $\mathrm{NC}$ patients (meaning high or low $[\mathrm{NA}]_{\text {plasma }}$ ).

In the present study, high $[\mathrm{NA}]_{\text {plasma }}$ in $\mathrm{AD}$ patients was associated with higher $\left[A \beta_{1-42}\right]_{C S F}$ than in other $A D$ patients with $[\mathrm{NA}]_{\text {plasma }}$ similar to NC patients. Several studies in the literature have compared $\left[\mathrm{A} \beta_{1-42}\right]_{\mathrm{CSF}}$ between MCI stage and more or less severe AD. MCI patients have been shown to have a higher $\left[\mathrm{A} \beta_{1-42}\right]_{\mathrm{CSF}}$ than AD patients ${ }^{34-37,40}$. There have also been conflicting 

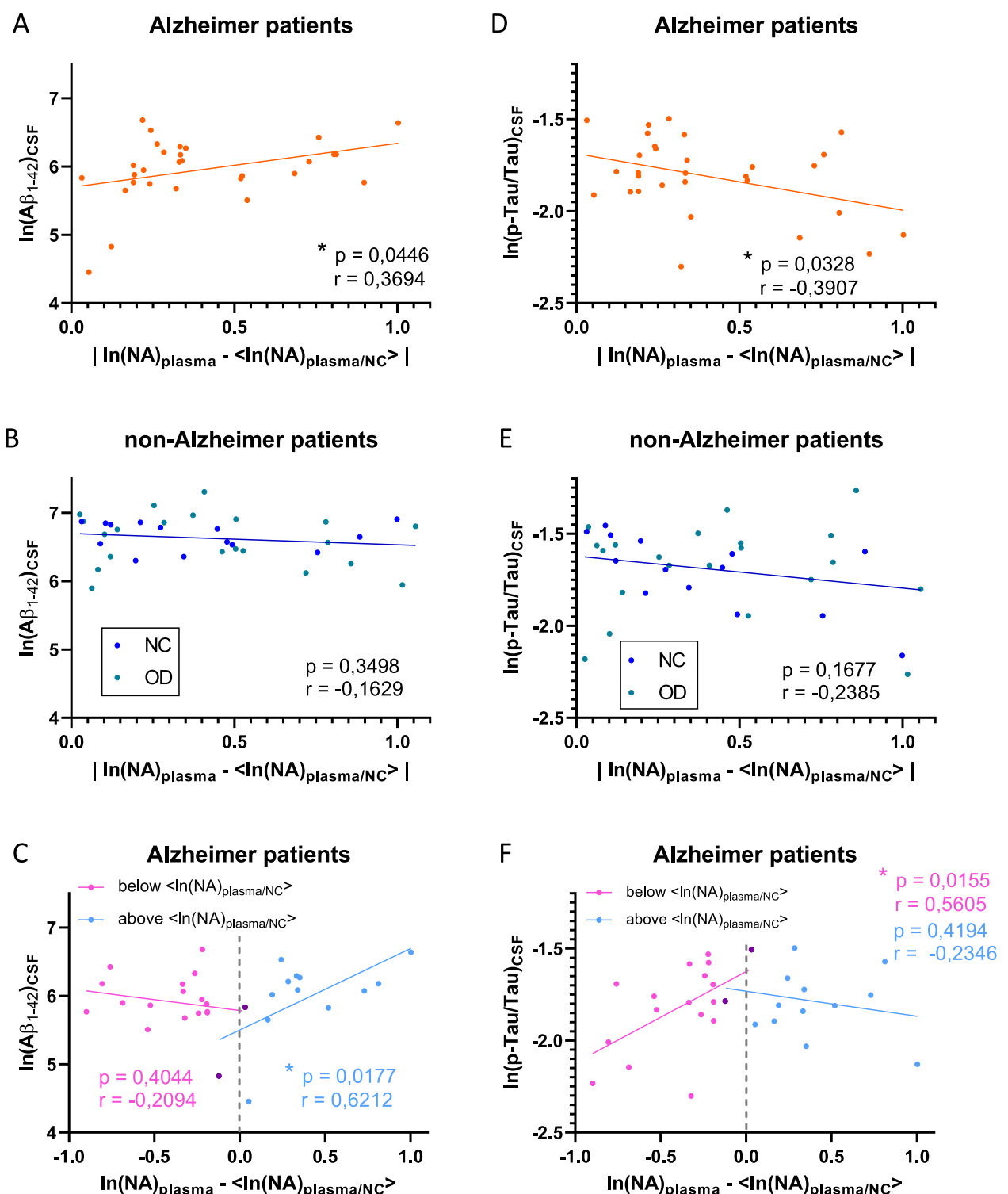

Fig. 3 Absolute $[N A]_{\text {plasma }}$ distance from median of NC patients correlates with $[A \beta 1-42]_{C S F}$ and $(p-T a u / T a u)_{C S F}$ in $A D$ patients. Linear correlations between absolute distance of $\operatorname{In}(\mathrm{NA})_{\text {plasma }}$ from median $\operatorname{In}(\mathrm{NA})$ plasma/NC with $\left[\mathrm{A} \beta_{1-42}\right]_{\mathrm{CSF}}$ in $\mathrm{AD}(\mathbf{a})$ and non- $\mathrm{AD}$ (b) patients. $\mathbf{c}$ Linear correlations between negative (in pink) and positive (in blue) values of $\ln (N A)_{\text {plasma }}-<\ln (N A)_{\text {plasma/NC }}>$ with $\left[A \beta_{1-42}\right]_{C S F}$ in $A D$ patients (points in purple were counted in both groups). Linear correlations between absolute distance of In(NA) plasma from median In(NA) plasma/NC with $(p-T a u / T a u)_{C S F}$ in $A D$ (d) and non-AD (e) patients. $\mathbf{f}$ Linear correlations between negative (in pink) and positive (in blue) values of $\ln (\mathrm{NA})_{\text {plasma }}{ }^{-}<\ln (\mathrm{NA})_{\text {plasma/NC }}>$ with $(p-T a u / T a u)_{C S F}$ in AD patients (points in purple were counted in both groups). * indicates $p$ value $<0.05$.

results concerning CSF Tau and p-Tau concentrations, showing no difference or increased levels in late-stage AD compared with $\mathrm{MCI}^{34-37,40,41,76}$. However, the differences observed were not always significant. The absence of statistical significance could be due to several parameters that differ among the different studies, such as the precision of the chemical dosage, sample storage, age of patients, sex ratio of the cohort groups, MMSE score, or advancement of the disease, involving stages of $\mathrm{AD}$ more or less distant from MCI. Moreover, the temporal evolution of biomarkers and cognitive markers of $\mathrm{AD}$ have previously been shown to differ according to disease stage (asymptomatic, MCI and dementia stage) ${ }^{77}$. We observed a lower $(\mathrm{p}-\mathrm{Tau} / \mathrm{Tau})_{\mathrm{CSF}}$ in $\mathrm{AD}$ patients with low $[\mathrm{NA}]_{\text {plasma }}$ than in non-AD patients with $[\mathrm{NA}]_{\text {plasma }}$ similar to $[\mathrm{NA}]_{\text {plasma }}$ in NC patients. A low (p-Tau/Tau $)_{\mathrm{CSF}}$ has also been associated with faster cognitive decline ${ }^{44}$, suggesting a possible link between this ratio and disease severity. In this way, low (p-Tau/ Tau) ${ }_{\mathrm{CSF}}$ associated with low MMSE score and low 
$\left[\mathrm{A} \beta_{1-42}\right]_{\mathrm{CSF}}$ is more likely to describe an advanced stage of $\mathrm{AD}$ pathology.

Regarding MMSE score, $\left[\mathrm{A} \beta_{1-42}\right]_{\mathrm{CSE}}$, and (p-Tau/ $\mathrm{Tau})_{\mathrm{CSF}}$, it is tempting to speculate that $\mathrm{AD}$ patients with high or low $[\mathrm{NA}]_{\text {plasma }}$ represent an early or late stage of $\mathrm{AD}$, setting $[\mathrm{NA}]_{\text {plasma }}$ as a potential marker of disease evolution. However, the link between the brain and $[\mathrm{NA}]_{\text {plasma }}$ alterations during $\mathrm{AD}$ is not yet understood. We hypothesize that the $[\mathrm{NA}]_{\text {plasma }}$ increase observed in $\mathrm{AD}$ patients could be due to (i) NA oversecretion in the CNS and disruption of the blood-brain barrier that may occur in $\mathrm{AD}^{78}$, (ii) release of cerebral NA in the blood through the CSF interface, with the potential of a linear correlation between $[\mathrm{NA}]_{\mathrm{CSF}}$ and $[\mathrm{NA}]_{\text {plasma }}{ }^{23}$, (iii) deregulation of NA secretion into the blood by the sympathetic system regulated by the $\mathrm{LC}^{26}$, and (iv) NA oversecretion from sympathetic noradrenergic postganglionic neurons to targeted organs and leakage or spillover into the blood ${ }^{79}$. We did not approach the concept of cognitive reserve in this article. Cognitive reserve is the phenomenon that maintains cognitive function despite advanced physiological symptoms of $\mathrm{AD}$ pathology in the brain ${ }^{80}$. Robertson developed the idea that LC-noradrenergic system activity may be the biological mechanism explaining the gap between cognitive function and pathophysiological observations of the disease (especially amyloid plaques) ${ }^{81}$, which has been further supported by several articles ${ }^{82-84}$. The pupillary response, a potential marker of NA-LC activity, illustrates that NA activity is enhanced by cognitively challenging stimuli. Knowing that NA seems to mediate neurocompensatory and neuroprotective effects, variables of cognitive reserve (education, enriched environment/ novelty, social interactions, mental activity) may lessen AD pathology through noradrenergic pathway activation ${ }^{81}$. Thus, this pathway represents a good candidate for the missing link in the cognition-brain pathology discrepancy observed in AD. Robertson has highlighted the fact that cognitive reserve phenomena through the action of NA in $\mathrm{AD}$ could have a direct effect on AD symptoms (antiinflammatory action, inhibition of amyloid aggregation, etc.) and/or a compensatory effect (increased connectivity, network reorganization). This complex question remains open and needs to be explored with further studies.

In this study, we showed for the first time that $[\mathrm{NA}]_{\text {plasma }}$ was correlated with MMSE score in the context of AD. We described particular profiles of MMSE score and CSF AD biomarkers for $\mathrm{AD}$ patients with high and low $[\mathrm{NA}]_{\text {plasma }}$. $[\mathrm{NA}]_{\text {plasma }}$ deregulation could hypothetically reflect overactivation and regression of the LC during AD. These results imply that (1) $[\mathrm{NA}]_{\text {plasma }}$ could support the early diagnosis of $\mathrm{AD}$, (2) $[\mathrm{NA}]_{\text {plasma }}$ could help to better characterize $\mathrm{AD}$ patient profiles during disease evolution, and
(3) the link between LC degeneration and $[\mathrm{NA}]_{\text {plasma }}$ in the context of $\mathrm{AD}$ needs to be further investigated. Combined with the MMSE score, $[\mathrm{NA}]_{\text {plasma }}$ could help the patient be referred to a specialist before the appearance of advanced $\mathrm{AD}$ symptoms. For example, physicians could advise patients with memory complaints, good MMSE scores and high $[\mathrm{NA}]_{\text {plasma }}$ to consult a neurologist for further examinations. Thus, this process could favor fast and early diagnosis of $\mathrm{AD}$, which opens new research potentials for blood biomarkers in $\mathrm{AD}$.

\section{Acknowledgements}

We also thank Claire Paquet, MD, PhD (Memory Resources and Research Center, Cognitive Neurology Center, INSERM UMR-S 942, University Hospital of Paris Diderot Saint Louis-Lariboisière-Fernand Widal, APHP, France) for providing the biological and clinical samples, as well as Jacques Callebert, PharmD, PhD (Department of Biochemistry, University Hospital of Paris Diderot Saint Louis-Lariboisière-Fernand Widal, APHP, France) for performing the blood sample analyses.

\section{Ethics approval and consent to participate}

Patients gave their informed and written consent to have their samples stored in an officially registered and ethically approved biological collection that has been approved by the Ethics Committee of Paris University Hospitals (CEERB [Comité d'Ethique En Recherche Biomédicale], Bichat University Hospital, Paris, France).

\section{Conflict of interest}

Alzohis is a company that has activities related to the submitted work. This study and this publication were produced and written in a responsible and ethical manner.

\section{Publisher's note}

Springer Nature remains neutral with regard to jurisdictional claims in published maps and institutional affiliations.

Received: 27 May 2019 Revised: 17 April 2020 Accepted: 22 April 2020 Published online: 03 July 2020

\section{References}

1. Motta, C. et al. Transcranial magnetic stimulation predicts cognitive decline in patients with Alzheimer's disease. J. Neurol. Neurosurg. Psychiatry 89, 1237-1242 (2018).

2. Blennow, K. A review of fluid biomarkers for Alzheimer's disease: moving from CSF to blood. Neurol. Ther. 6, 15-24 (2017).

3. Carswell, C. J. et al. Clinical utility of amyloid PET imaging with (18)F-florbetapir: a retrospective study of 100 patients. J. Neurol. Neurosurg. Psychiatry $\mathbf{8 9}$, 294-299 (2018).

4. Jack, C. R. et al. NIA-AA research framework: toward a biological definition of Alzheimer's disease. Alzheimer's Dement. J. Alzheimer's Assoc. 14, 535-562 (2018).

5. Paterson, R. W. et al. Cerebrospinal fluid in the differential diagnosis of Alzheimer's disease: clinical utility of an extended panel of biomarkers in a specialist cognitive clinic. Alzheimer's Res. Ther. 10, 32 (2018).

6. Gannon, M. et al. Noradrenergic dysfunction in Alzheimera's disease. Front. Neurosci. 9, 220 (2015).

7. Trillo, L. et al. Ascending monoaminergic systems alterations in Alzheimer's disease. Translating basic science into clinical care. Neurosci. Biobehav. Rev. 37, 1363-1379 (2013).

8. Kelly, S. C. et al. Locus coeruleus cellular and molecular pathology during the progression of Alzheimer's disease. Acta Neuropathol. Commun. 5, 8 (2017).

9. Sara, S. J. The locus coeruleus and noradrenergic modulation of cognition. Nat. Rev. Neurosci. 10, 211-223 (2009).

10. Satoh, A. \& lijima, K. M. Roles of tau pathology in the locus coeruleus (LC) in age-associated pathophysiology and Alzheimer's disease pathogenesis: potential strategies to protect the LC against aging. Brain Res. 1702, 17-28 (2019). 
11. Grudzien, A. et al. Locus coeruleus neurofibrillary degeneration in aging, mild cognitive impairment and early Alzheimer's disease. Neurobiol. Aging $\mathbf{2 8}$ 327-335 (2007).

12. Olivieri, P. et al. Early alteration of the locus coeruleus in phenotypic variants of Alzheimer's disease. Ann. Clin. Transl. Neurol. 6, 1345-1351 (2019).

13. Takahashi, J. et al. Detection of changes in the locus coeruleus in patients with mild cognitive impairment and Alzheimer's disease: high-resolution fast spinecho T1-weighted imaging. Geriatr. Gerontol. Int. 15, 334-340 (2015).

14. Ghosh, A. et al. An experimental model of Braak's pretangle proposal for the origin of Alzheimer's disease: the role of locus coeruleus in early symptom development. Alzheimer's Res. Ther. 11, 59 (2019).

15. Giorgi, F. S. et al. The neuroanatomy of the reticular nucleus locus coeruleus in Alzheimera's Disease. Front. Neuroanat. 11, 80 (2017).

16. Betts, M. J. et al. Locus coeruleus imaging as a biomarker for noradrenergic dysfunction in neurodegenerative diseases. Brain 142, 2558-2571 (2019).

17. Theofilas, P. et al. Locus coeruleus volume and cell population changes during Alzheimer's disease progression: a stereological study in human postmortem brains with potential implication for early-stage biomarker discovery. Alzheimer's Dement. J. Alzheimer's Assoc. 13, 236-246 (2017).

18. Ross, J. A., Gliebus, G. \& Van Bockstaele, E. J. Stress induced neural reorganization: a conceptual framework linking depression and Alzheimer's disease. Prog. Neuropsychopharmacol. Biol. Psychiatry 85, 136-151 (2018).

19. Ross, J. A., McGonigle, P. \& Van Bockstaele, E. J. Locus Coeruleus, norepinephrine and A $\beta$ peptides in Alzheimer's disease. Neurobiol. Stress 2, 73-84 (2015).

20. Adolfsson, R., Gottfries, C., Roos, B. \& Winblad, B. Changes in the brain catecholamines in patients with dementia of Alzheimer type. Br. J. Psychiatry 135, 216-223 (1979).

21. Vermeiren, Y., Van Dam, D., Aerts, T., Engelborghs, S. \& De Deyn, P. P. Monoaminergic neurotransmitter alterations in postmortem brain regions of depressed and aggressive patients with Alzheimer's disease. Neurobiol. Aging 35, 2691-2700 (2014).

22. Elrod, R. et al. Effects of Alzheimer's disease severity on cerebrospinal fluid norepinephrine concentration. Am. J. Psychiatry 154, 25-30 (1997).

23. Raskind, M. A., Peskind, E. R., Halter, J. B. \& Jimerson, D. C. Norepinephrine and MHPG levels in CSF and plasma in Alzheimer's disease. Arch. Gen. Psychiatry 41, 343 (1984).

24. Kalinin, S. et al. Noradrenaline deficiency in brain increases $\beta$-amyloid plaque burden in an animal model of Alzheimer's disease. Neurobiol. Aging $\mathbf{2 8}$ 1206-1214 (2007).

25. Gordan, R., Gwathmey, J. K. \& Xie, L.H. Autonomic and endocrine control of cardiovascular function. World J. Cardiol. 7, 204-214 (2015).

26. Szabadi, E. Functional neuroanatomy of the central noradrenergic system. J. Psychopharmacol. (Oxf.) 27, 659-693 (2013).

27. Wehrwein, E. A., Orer, H. S. \& Barman, S. M. Overview of the anatomy, physiology, and pharmacology of the autonomic nervous system. Compr. Physiol. 6, 1239-1278 (2016)

28. Umegaki, $H$. et al. Low plasma epinephrine in elderly female subjects of dementia of Alzheimer type. Brain Res. 858, 67-70 (2000)

29. Umegaki, H., Tamaya, N., Shinkai, T. \& Iguchi, A. The metabolism of plasma glucose and catecholamines in Alzheimer's disease. Exp. Gerontol. 35, 1373-1382 (2000).

30. Petrie, E. C., Peskind, E. R., Dobie, D. J., Veith, R. C. \& Raskind, M. A. Plasma catecholamine and cardiovascular responses to physostigmine in Alzheimer's disease and aging. Psychoneuroendocrinology 26, 147-164 (2001).

31. Reisberg, B. et al. Stage-specific behavioral, cognitive, and in vivo changes in community residing subjects with age-associated memory impairment and primary degenerative dementia of the Alzheimer type. Drug Dev. Res. 15, 101-114 (1988)

32. Albert, M. S. et al. The diagnosis of mild cognitive impairment due to Alzheimer's disease: recommendations from the National Institute on AgingAlzheimer's Association workgroups on diagnostic guidelines for Alzheimer's disease. Alzheimer's Dement. J. Alzheimer's Assoc. 7, 270-279 (2011).

33. Vega, J. N. \& Newhouse, P. A. Mild cognitive impairment: diagnosis, longitudinal course, and emerging treatments. Curr. Psychiatry Rep. 16, 490 (2014).

34. Poulsen, K., Bahl, J. M., Simonsen, A. H., Hasselbalch, S. G. \& Heegaard, N. H. Distinct transthyretin oxidation isoform profile in spinal fluid from patients with Alzheimer's disease and mild cognitive impairment. Clin. Proteom. 11, 12 (2014).

35. Janelidze, S. et al. CSF biomarkers of neuroinflammation and cerebrovascular dysfunction in early Alzheimer disease. Neurology 91, e867-e877 (2018).
36. Hampel, $H$. et al. Value of CSF beta-amyloid1-42 and tau as predictors of Alzheimer's disease in patients with mild cognitive impairment. Mol. Psychiatry 9, 705-710 (2004).

37. Höglund, K. et al. Prediction of Alzheimer's disease using a cerebrospinal fluid pattern of C-terminally truncated beta-amyloid peptides. Neurodegener. Dis. $\mathbf{5}$, 268-276 (2008)

38. Han, P. et al. A quantitative analysis of brain soluble tau and the tau secretion factor. J. Neuropathol. Exp. Neurol. 76, 44-51 (2017).

39. Schönknecht, P., Pantel, J., Kaiser, E. \& Schröder, J. Total and phospho-tau (Thr 181) CSF levels in patients with mild cognitive impairment and Alzheimer's disease. Psychogeriatra Pol. 1, 185-192 (2004).

40. Wildsmith, K. R. et al. Identification of longitudinally dynamic biomarkers in Alzheimer's disease cerebrospinal fluid by targeted proteomics. Mol. Neurodegener. 9, 22 (2014).

41. Riemenschneider, M. et al. Phospho-tau/total tau ratio in cerebrospinal fluid discriminates Creutzfeldt-Jakob disease from other dementias. Mol. Psychiatry 8, 343-347 (2003)

42. Corrêa, J. D., Starling, D., Teixeira, A. L., Caramelli, P. \& Silva, T. A. Chemokines in CSF of Alzheimer's disease patients. Arq. Neuropsiquiatr. 69, 455-459 (2011).

43. Pijnenburg, Y. A. L. et al. CSF neurofilaments in frontotemporal dementia compared with early onset Alzheimer's disease and controls. Dement. Geriatr. Cogn. Disord. 23, 225-230 (2007).

44. Kester, M. I. et al. CSF biomarkers predict rate of cognitive decline in Alzheimer disease. Neurology 73, 1353-1358 (2009).

45. Teunissen, $C$. E. et al. Plasma amyloid- $\beta$ (A $\beta 42)$ correlates with cerebrospinal fluid Aß42 in Alzheimer's disease. J. Alzheimer's Dis. 62, 1857-1863 (2018).

46. Nakamura, A. et al. High performance plasma amyloid- $\beta$ biomarkers for Alzheimer's disease. Nature 554, 249-254 (2018)

47. Giedraitis, V. et al. The normal equilibrium between CSF and plasma amyloid beta levels is disrupted in Alzheimer's disease. Neurosci. Lett. 427, 127-131 (2007).

48. Park, J.-C. et al. Chemically treated plasma $A B$ is a potential blood-based biomarker for screening cerebral amyloid deposition. Alzheimer's Res. Ther. $\mathbf{9}$, 20 (2017).

49. Lövheim, $\mathrm{H}$. et al. Plasma concentrations of free amyloid $\beta$ cannot predict the development of Alzheimer's disease. Alzheimer's Dement. J. Alzheimer's Assoc. 13, 778-782 (2017).

50. Figurski, M. J. et al. Improved protocol for measurement of plasma $\beta$-amyloid in longitudinal evaluation of Alzheimer's Disease neuroimaging Initiative study patients. Alzheimer's Dement. J. Alzheimer's Assoc. 8, 250-260 (2012).

51. Poljak, A. \& Sachdev, P. S. Plasma amyloid beta peptides: an Alzheimer's conundrum or a more accessible Alzheimer's biomarker? Expert Rev. Neurother. 17, 3-5 (2017).

52. Takeda, S., Sato, N., Rakugi, H. \& Morishita, R. Plasma $\beta$-amyloid as potential biomarker of Alzheimer disease: possibility of diagnostic tool for Alzheimer disease. Mol. Biosyst. 6, 1760 (2010).

53. Kim, H. J. et al. Elevation of the plasma $A \beta 40 / A \beta 42$ ratio as a diagnostic marker of sporadic early-onset Alzheimer's disease. J. Alzheimer's Dis. 48, 1043-1050 (2015).

54. Yang, C.-C. et al. Assay of plasma phosphorylated tau protein (Threonine 181) and total tau protein in early-stage Alzheimer's disease. J. Alzheimer's Dis. 61 1323-1332 (2018).

55. Rembach, A. et al. Changes in plasma amyloid beta in a longitudinal study of aging and Alzheimer's disease. Alzheimer's Dement. 10, 53-61 (2014).

56. Le Bastard, N. et al. No correlation between time-linked plasma and CSF A levels. Neurochem. Int. 55, 820-825 (2009).

57. Fossati, S. et al. Plasma tau complements CSF tau and P-tau in the diagnosis of Alzheimer's disease. Alzheimer's Dement. Amst. Neth. 11, 483-492 (2019).

58. Janelidze, S. et al. Plasma $\beta$-amyloid in Alzheimer's disease and vascular disease. Sci. Rep. 6, 26801 (2016).

59. Fagan, A. M. et al. Comparison of analytical platforms for cerebrospinal fluid measures of $\beta$-amyloid 1-42, total tau, and p-tau181 for identifying Alzheimer disease amyloid plaque pathology. Arch. Neurol. 68, 1137 (2011).

60. Xin, S.-H., Tan, L., Cao, X., Yu, J.-T. \& Tan, L. Clearance of amyloid beta and tau in Alzheimer's Disease: from mechanisms to therapy. Neurotox. Res. 34, 733-748 (2018).

61. Folstein, M. F., Folstein, S. E. \& McHugh, P. R. Mini-mental state'. A practical method for grading the cognitive state of patients for the clinician. J. Psychiatr. Res. 12, 189-198 (1975). 
62. Tsoi, K. K. F., Chan, J. Y. C., Hirai, H. W., Wong, S. Y. S. \& Kwok, T. C. Y. Cognitive tests to detect dementia: a systematic review and meta-analysis. JAMA Intern. Med. 175, 1450 (2015).

63. Legdeur, N. et al. Cognitive functioning of individuals aged 90 years and older without dementia: a systematic review. Ageing Res. Rev. 36, 42-49 (2017).

64. Jia, J. et al. Efficacy and safety of Donepezil in Chinese patients with severe alzheimer's disease: a randomized controlled trial. J. Alzheimer's Dis. 56, 1495-1504 (2017).

65. Yoelin, A. B. \& Saunders, N. W. Score disparity between the MMSE and the SLUMS. Am. J. Alzheimer's Dis. Dementiasr. 32, 282-288 (2017).

66. Harrison, J. Cognitive approaches to early Alzheimer's Disease diagnosis. Med. Clin. North Am. 97, 425-438 (2013)

67. Doody, R. S., Massman, P. \& Dunn, J. K. A method for estimating progression rates in Alzheimer Disease. Arch. Neurol. 58, 449-454 (2001).

68. Cleophas, T. J. \& Zwinderman, A. H. Logarithmic transformations, a great help to statistical analyses. In Clinical Data Analysis on a Pocket Calculator: Understanding the Scientific Methods of Statistical Reasoning and Hypothesis Testing (eds. Cleophas, T. J. \& Zwinderman, A. H.) 243-247 (Springer International Publishing, 2016).

69. LV, C. et al. Determination of catecholamines and their metabolites in rat urine by ultra-performance liquid chromatography-tandem mass spectrometry for the study of identifying potential markers for Alzheimer's disease. J. Mass Spectrom. 50, (2015).

70. Francis, B. M. et al. Reduced tissue levels of noradrenaline are associated with behavioral phenotypes of the TgCRND8 mouse model of Alzheimer's Disease. Neuropsychopharmacology 37, 1934-1944 (2012).

71. Von Linstow, C. U. et al. Effect of aging and Alzheimer's disease-like pathology on brain monoamines in mice. Neurochem. Int. 108, 238-245 (2017).

72. Rorabaugh, J. M. et al. Chemogenetic locus coeruleus activation restores reversal learning in a rat model of Alzheimer's disease. Brain 140, 3023-3038 (2017).
73. Liu, L. et al. Simultaneous determination of catecholamines and their metabolites related to Alzheimer's disease in human urine. J. Sep. Sci. 34, 1198-1204 (2011).

74. Vermeiren, Y. et al. Brain serotonergic and noradrenergic deficiencies in behavioral variant frontotemporal dementia compared to early-onset Alzheimer's Disease. J. Alzheimer's Dis. 53, 1079-1096 (2016)

75. Heneka, M. T. et al. Locus ceruleus controls Alzheimer's disease pathology by modulating microglial functions through norepinephrine. Proc. Natl Acad. Sci. USA. 107, 6058-6063 (2010).

76. Betts, M. J. et al. Locus coeruleus MRI contrast is reduced in Alzheimer's disease dementia and correlates with CSF A $\beta$ levels. Alzheimer's Dement. Amst. Neth. 11, 281-285 (2019)

77. Bertens, D., Knol, D. L., Scheltens, P. \& Visser, P. J. Temporal evolution of biomarkers and cognitive markers in the asymptomatic, $\mathrm{MCl}$ and dementia stage of Alzheimer's disease. Alzheimer's Dement. J. Alzheimer's Assoc. 11, 511-522 (2015)

78. Yamazaki, Y. \& Kanekiyo, T. Blood-brain barrier dysfunction and the pathogenesis of Alzheimer's Disease. Int. J. Mol. Sci. 18, (2017).

79. Goldstein, D. S., Eisenhofer, G. \& Kopin, I. J. Sources and significance of plasma levels of catechols and their metabolites in humans. J. Pharmacol. Exp. Ther. 305, 800-811 (2003).

80. Stern, Y. \& Barulli, D. Cognitive reserve. Neuropsychologia 47, 2015-2028 (2009).

81. Robertson, I. H. A noradrenergic theory of cognitive reserve: implications for Alzheimer's disease. Neurobiol. Aging 34, 298-308 (2013).

82. Wilson, R. S. et al. Neural reserve, neuronal density in the locus ceruleus, and cognitive decline. Neurology 80, 1202-1208 (2013).

83. $\mathrm{Xu}, \mathrm{W} ., \mathrm{Yu}, \mathrm{J} .-\mathrm{T} ., \mathrm{Tan}, \mathrm{M} . \mathrm{S}$. \& Tan, L. Cognitive reserve and Alzheimer's Disease. Mol. Neurobiol. 51, 187-208 (2015).

84. Fleck, J. I. et al. The impact of age and cognitive reserve on resting-state brain connectivity. Front. Aging Neurosci. 9, (2017) 